Mathématiques et sciences humaines
Mathematics and social sciences

184 | Hiver 2008

Varia

\title{
Marc Barbut, La mesure des inégalités. Ambiguités et paradoxes, Genève-Paris, Librairie Droz, 2007.
}

Marc Barbut, La mesure des inégalités. Ambiguités et paradoxes, Genève-Paris, Librairie Droz, 2007.

\section{Bernard Bru}

\section{OpenEdition}

\section{Journals}

Édition électronique

URL : http://journals.openedition.org/msh/10986

DOI : $10.4000 / \mathrm{msh} .10986$

ISSN : 1950-6821

Éditeur

Centre d'analyse et de mathématique sociales de l'EHESS

Édition imprimée

Date de publication : 31 décembre 2008

Pagination : 113-114

ISSN : 0987-6936

Référence électronique

Bernard Bru, «Marc Barbut, La mesure des inégalités. Ambiguïtés et paradoxes, Genève-Paris, Librairie Droz, 2007. ", Mathématiques et sciences humaines [En ligne], 184 | Hiver 2008, mis en ligne le 25 février 2009, consulté le 25 septembre 2020. URL : http://journals.openedition.org/msh/10986 ; DOI : https:// doi.org/10.4000/msh.10986

(c) École des hautes études en sciences sociales 
Marc Barbut, La mesure des inégalités. Ambiguïtés et paradoxes, Genève-Paris, Librairie Droz, 2007.

À la demande de Giovanni Busino, éditeur des œuvres de Pareto, qui fut longtemps titulaire de la chaire de sociologie de l'Université de Lausanne, qu'occupa Pareto, Marc Barbut a réuni dans ce beau livre un choix de quelques-uns de ses articles sur la modélisation mathématique des inégalités économiques et sociales. Plus encore qu'un recueil d'articles, tous originaux et d'une grande richesse, il s'agit d'un véritable cours sur «la mesure des inégalités », sans équivalent dans la littérature actuelle, un cours complet qui s'adresse bien sûr aux étudiants économistes ou sociologues, mais qui devrait intéresser aussi bien et parfois plus encore les statisticiens, mathématiciens, économistes, sociologues, historiens des mathématiques ou de la pensée économique, les journalistes politiques et économiques, les professeurs de lycée, ..., et tous ceux qui, un jour ou l'autre, se sont demandé si notre société était plus inégalitaire qu'avant et ce qu'il faudrait faire pour améliorer les choses, c'est-à-dire tout le monde. Et le livre de Marc Barbut est ainsi rédigé qu'il peut se lire sans aucune connaissance mathématique particulière, tout en allant suffisamment au fond mathématique des choses, pour qu'un lecteur mathématicien y trouve une ample moisson qu'il ne trouverait nulle part ailleurs. Un livre unique, nécessaire, indispensable.

Qu'un tel livre soit indispensable, il n'est pas difficile de s'en convaincre. Il suffit par exemple de consulter Internet, où l'on nous présente, le plus sérieusement du monde, un classement par ordre d'inégalités de revenus de tous les pays du monde (site Wikipedia articles «Coefficient de Gini » et «Liste des pays par égalité de revenus ») dans lequel on apprend que le coefficient de Gini de la France est de 0,327, ce qui la place à la $34^{\mathrm{e}}$ place mondiale entre le Tadjikistan et le Pakistan, résultat intéressant qui conforte sans doute l'une des thèses les mieux argumentées du livre de Marc Barbut, « il n'y a pas de mesure de l'inégalité », et l'indice de Gini, pour remarquable qu'il soit, ne saurait en tenir lieu universellement. Comme le montre Marc Barbut, Gini lui-même le savait fort bien puisque son « indice » n'est qu'un paramètre possible de la « courbe de Gini » qui tient compte de la complexité des situations particulières et qui sans doute manifesterait quelques différences entre le Tadjikistan, la France et le Pakistan, pourtant d'indices de Gini égaux à fort peu près.

Le cours de Marc Barbut est divisé en deux parties. La première partie est «nonparamétrique ». Elle s'intitule «Inégalités et concentration. Paradoxes ». Marc Barbut montre sur des données réelles judicieusement choisies, l'intérêt et les limites des courbes de concentration de Gini et Lorenz et examine avec beaucoup de finesse les « paradoxes» réels ou apparents de ce type de représentation. Cette partie se termine par une comparaison entièrement originale, à notre connaissance, entre les fonctions de concentration de Gini et celles introduites par Paul Lévy en 1931, qui en a fait un outil puissant pour l'étude des sommes de variables aléatoires indépendantes, sans d'ailleurs que ni Gini, ni Lorenz, ni Lévy ne se rendent compte qu'ils manipulaient des objets analogues. La seconde partie est «paramétrique » et suppose que les distributions sont de type Pareto, de sorte que le paramètre de Pareto qui figure en exposant peut tenir lieu d'indice naturel d'inégalité. Cette partie s'intitule «Lois de V. Pareto et de P. Lévy. Ambiguïtés ». Elle entend en effet montrer que la généralité des lois de Pareto peut se comprendre assez naturellement dans le cadre de la théorie des lois stables de Lévy et de leurs domaines d'attraction. Là encore ni Vilfredo Pareto, ni Paul Lévy ne se sont rendu compte qu'ils traitaient d'objets comparables et que la théorie de Lévy était l'analogue pour les lois de Pareto de la théorie laplacienne pour la loi normale. Un tel 
rapprochement ne semble avoir été fait qu'à la fin des années cinquantes par Benoit Mandelbrot et Georges Th. Guilbaud, dans le cadre du séminaire Guilbaud et si Mandelbrot se fit dès lors le prophète parétien du monde anglo-saxon, imposant en économie la «loi de Pareto-Lévy », il ne faut pas oublier que, dans le même temps, Guilbaud, Barbut et quelques très rares autres tentèrent de convaincre la statistique française de l'universalité des statistiques parétiennes, si bien que ce livre est aussi une pièce historique importante pour qui voudrait faire l'histoire de la statistique parisienne (et parétienne) de la seconde moitié du $20^{\mathrm{e}}$ siècle.

Marc Barbut fait également une place dans cette histoire à Maurice Fréchet, pour deux raisons. D'abord parce que Fréchet semble avoir été le premier à remarquer cette propriété importante des lois de Pareto que la moyenne des valeurs supérieures à une valeur donnée $x$ est proportionnelle à $x$, le coefficient de proportionnalité étant le conjugué du paramètre de Pareto, un autre indice d'inégalité. Ensuite parce que Fréchet a montré que les lois de Pareto-Lévy peuvent être vues également comme les lois asymptotiques des maximum d'un grand nombre de variables indépendantes et qu'elles sont ainsi pour l'extrême ce que la loi normale est pour la moyenne. "L'homme de Pareto est l'homme des valeurs extrêmes », tandis que l'homme normal est un homme moyen.

À côté des propriétés mathématiques fondamentales des lois de Pareto-Lévy que Marc Barbut expose avec le recul et la clarté limpide que donnent des années d'enseignement et de recherche au CAMS et ailleurs, l'auteur expose avec lucidité les ambiguités des travaux de Pareto sur les distributions de revenus, et sa querelle « idéologique » plus que mathématique avec Georges Sorel. Il y a là une page d'histoire peu connue et riche d'enseignements actuels sur le rôle du hasard et de la nécessité dans les inégalités de revenus ou de ce qu'on voudra.

Marc Barbut donne évidemment de multiples exemples de lois de Pareto dans tous les domaines possibles, économie, géographie, linguistique, etc. Ajoutons en un dû à G. Th. Guilbaud, dans un de ses derniers écrits statistiques :

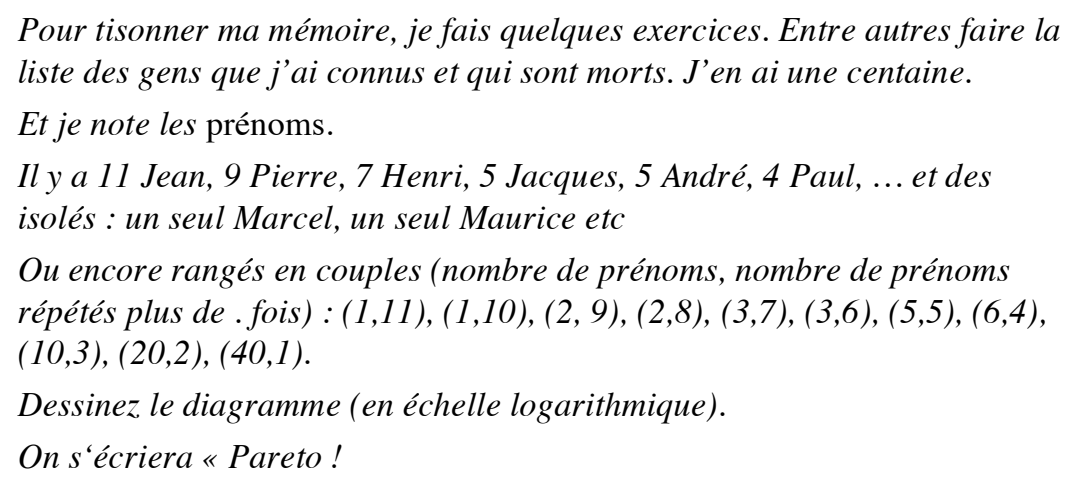

